\title{
How can the Quality of Medical Data in Pharmacovigilance, Pharmacoepidemiology and Clinical Studies be Guaranteed?
}

François Alla ${ }^{1}$, Myriam Rosilio ${ }^{2}$, Christian Funck-Brentano ${ }^{3}$, and participants of round table $N^{\circ} 2$ of Giens Workshops XXVIII: Philippe Barthélémy ${ }^{4}$, Sophie Brisset ${ }^{5}$, Dominic Cellier ${ }^{6}$, Olivier Chassany ${ }^{7}$, Jean-Paul Demarez ${ }^{8}$, Vincent Diebolt ${ }^{9}$, Alain Francillon ${ }^{10}$, Laetitia Gambotti ${ }^{11}$, Hakima Hannachi ${ }^{12}$, Philippe Lechat ${ }^{13}$, François Lemaire ${ }^{14}$, Michel Lièvre ${ }^{15}$, Christophe Misse ${ }^{16}$, Marina Nguon ${ }^{17}$, Antoine Pariente ${ }^{18}$, Michel Rosenheim ${ }^{19}$ and Nadine Weisslinger-Darmon ${ }^{20}{ }^{\dagger}$

1 Université de Lorraine, Nancy, France

2 Laboratoire Lilly France, Neuilly-sur-Seine, France

3 Université Pierre et Marie Curie, Paris, France

4 Laboratoire AstraZeneca, Rueil-Malmaison, France

5 Laboratoire Ax-Pharma, Paris, France

6 Laboratoire Merck Serono, Lyon, France

DRCD, Paris, France

8 Laboratoire Pierre Fabre Médicament, Castres, France

9 F-CRIN, Toulouse, France

10 Laboratoire Novartis, Rueil-Malmaison, France

11 URC Pitié-Salpétrière, AP-HP, Paris, France

12 Laboratoire Merck Sharp Dohme, Courbevoie, France

13 Université Paris 7, Paris, France

14 DRCD Île-de-France, Paris, France

15 Pharmacologie clinique, CHU, Lyon, France

16 DRCD, Paris, France

17 DRCD, Pôle vigilance, Paris, France

18 Centre régional de Pharmacovigilance, $\mathrm{CHU}$, Bordeaux, France

19 Assistance Publique - Hôpitaux de Paris, Paris, France

20 Laboratoire GlaxoSmithKline, Marly-le-Roi, France

Text received March $15^{\text {th }}, 2013$; accepted May $7^{\text {th }}, 2013$

\section{Keywords:}

quality; data;

guidelines;

clinical trials;

pharmacoepidemiology;

pharmacovigilance
Abstract-The development of medicinal products is subject to quality standards aimed at guaranteeing that database contents accurately reflect the source documents. Paradoxically, these standards hardly address the quality of the source data itself. The objective of this work was to propose recommendations to improve data quality in three fields (pharmacovigilance, pharmacoepidemiology and clinical studies). The analysis was focused on the data and on the critical stages presenting critical quality problems, for which the current guidelines are insufficiently detailed, unsuitable and/or poorly applied. Finally, recommendations have been proposed, mainly focused on the origin of the data and its transcription.

Abbreviations: see end of article.

\footnotetext{
$\dagger$ Articles, analyzes and proposals from the Giens workshops are those of the authors and do not prejudice the position of their parent organization.
} 


\section{Introduction}

The development of medicinal products has been subject to very strict quality standards for many years, such as international conference of harmonization (ICH) guidelines for clinical trials. ${ }^{[1]}$ Generally, the purpose of these guidelines and procedures is to guarantee that database contents accurately reflect the source file. However, they hardly address, if at all, the nevertheless essential issue of the quality of the source data. As an example, the evaluation of critical events during a clinical trial has shown that the interpretations of investigator physicians are, at the very least, questionable. Several studies have demonstrated that the classification proposed by the investigators for major cardiovascular events were changed by an independent critical events adjudication committee in a quarter to a third of cases. ${ }^{[2,3,4]}$ The poor quality of medical information contained in the source data could change the conclusion of a trial. Similarly, the lack of quality of medical information contained in pharmacoepidemiology or pharmacovigilance data could have serious consequences. Apart from patient security and public health, risks related to the absence of data quality and to the diffusion and use of nonvalidated information are of a decision-making nature (e.g. making an inadequate regulatory decision), are social and media risks (there are many recent examples) as well as scientific risks (i.e. poor quality data could lead to false hypotheses being drawn up which would require time and resources to refute). This information risk has become a concern for the regulator, as illustrated by the proposed European regulation relating to clinical trials with the objective to avoid two distinct risks: "[...] the risk for patients safety and the risk for data reliability". ${ }^{[5]}$ Interest in the quality of medical information generated by the source data is therefore a major issue, and yet, it has been relatively rarely covered in the literature.

The objective of this work was to propose recommendations to improve the data quality, and more precisely the "medical validity" of data in three types of studies: pharmacovigilance, pharmacoepidemiology and clinical studies. The approach was based on the exchange of observations, experiences and expertise of those involved in these three fields. The work is focused on data quality and has not addressed data use (e.g. statistical analysis). The proposals exposed at the end of this article have been formulated following a structured approach, the elements of which are presented in this article: 1) definition of the framework, in particular of the data and its sources, 2) description of the process and 3) demonstration of critical points, and then 4) analysis of the existing guidelines and their limits.

\section{Framework, typology and definitions}

This work relates to medical data and information used in three main fields concerning the evaluation and security of medicinal products: clinical trials, pharmacoepidemiology and pharmacovigilance, defined as follows:
1. The field of phase III clinical studies: phase III clinical trials evaluate the benefit/risk ratio of a future medicinal product, especially by assessing its medium to long-term safety and efficacy. Quality controls and audits are frequently carried out on these studies, particularly when they are registration trials for marketing authorization (MA).

2. The field of pharmacoepidemiology is the study of the use and effects of medicinal products on large populations in order to describe and analyze the practices and conditions of use, evaluate the security and efficacy as an alternative to a clinical trial, evaluate the effectiveness in a routine situation ([comparative] effectiveness research), carry out economic and medico-economic evaluations. ${ }^{[6]}$ Pharmacoepidemiology can be in a regulatory context (e.g. post-reimbursement studies; post marketing studies).

3. The field of pharmacovigilance: pharmacovigilance is the surveillance to assess risk of adverse effects resulting from the use of drug products, plus the analysis and management of these risks. Within the framework of clinical trials, pharmacovigilance plays an important role since by definition the safety of a medicinal product under study is evaluated during these trials. Special attention is paid to suspected unexpected serious adverse reaction (SUSAR), which are serious adverse reactions that are unexpected or unintended responses related to an experimental drug.

\subsection{What data?}

All data contributing to study objectives are targeted by this analysis. In particular, data describing and providing elements to verify health states and events, histories and other clinical and behavioral characteristics of patients, the diagnostic procedures and results, treatments and interventions, compliance and persistence, etc. These data can be used for the selection and definition of the population, as well as for exposure factors, adjustment factors and/ or endpoint criteria.

\subsection{What sources?}

Some sources are more specific in some fields. Clinical trials are generally based on data specifically collected and/or generated during the treatment. As for pharmacoepidemiology, it involves the exploitation of medico-administrative data. Finally, pharmacovigilance classically uses data from reporting, either coming from clinical trials or from collated, unsolicited reports, sometimes in a context of prospective follow-up for a risk management plan.

There are potentially many sources of this data:

- data collected (or so-called "prospective" data): assessments performed for the study (clinical examinations, questionnaires, complementary assessments, etc.);

- data, specifically taken from medical records, generated during the standard patients follow-up (or so-called "retrospective" 
Table I. Description of the fields and sources identified.

\begin{tabular}{lcc}
\hline Fields & Phase III clinical trial & Pharmacovigilance \\
Sources & $\mathrm{xxx}$ & $\mathrm{x} / \mathrm{xx}$ \\
\hline Specific data & $\mathrm{xx}$ & $\mathrm{xx}$ \\
Retrospective data & $\mathrm{xx}$ & $\mathrm{xxx}$ \\
Declarations & $\mathrm{x}$ & $\mathrm{xx}$ \\
Medico-administrative data & $\mathrm{x}$ & $\mathrm{xx}$ \\
\hline
\end{tabular}

$\mathrm{x}$ : data rarely used in the mentioned field;

$\mathrm{xx}$ : data occasionally used in the mentioned field;

$\mathrm{xxx}$ : data frequently used in the mentioned field.

data): assessments performed in the context of patient care and then re-used for the study;

- medico-administrative data: data initially collected for care management and reimbursement purposes. They can subsequently be used in the aim of public health or research. In France, the most frequently used are data from the French national discharge database (programme de médicalisation des systèmes d'information [PMSI]) and French national Health insurance information system (système national d'informations inter régions d'Assurance maladie [SNIIRAM]);

- pharmacovigilance data: data obtained within the context of safety follow-up of medicinal products tested in clinical trials (reporting of non serious, serious and unexpected adverse events) as well as within the context of the national pharmacovigilance system (unsolicited reports on marketed medicinal products).

Given these specificities, the current guidelines are generally segmented and do not cover all the possible sources for a given use. However, the uses are becoming more diversified and each of these fields is now likely to use each of the sources or even a combination of them (table I).

For this reason and because of the similarity of the problems, all three fields were analyzed together.

\subsection{From data to information}

Firstly, - and very importantly - the concept of "data" (raw element directly collected by assessments or generated from data collected for a different purpose) must be distinguished from the notion of "information", which results from processing and interpreting the raw data. For example, weight and height are data, whereas the presence or not of obesity, as defined by a combination of these data to which a threshold is applied, is information. Quality data can give rise to poor quality information depending on the processing and interpretation. It is particularly important to consider this distinction within the context of the use of medico-administrative data. In fact, these data are generally quality data (e.g. information in a reimbursement database "prescription of anti-hypertension treatment" corresponds to reality). However, deducing from the prescription of an antihypertension treatment that the patient has hypertension is a potentially problematic interpretation (e.g. by lack of treatment specificity: diuretics can be prescribed for hypertension but also for heart failure).

\subsection{What is quality data?}

Quality data could be defined as data that correctly represents reality. This is the most natural definition but is hampered by the perception of "absolute" quality for which more is always better. This absolute is nevertheless not conceivable nor desirable: for example what is the use of having a patient's weight to the nearest gram? The level of optimal quality depends on the objective: for example if the standard for assessment of left ventricular mass is magnetic resonnance imaging (MRI), an assessment by echocardiography could be considered as sufficient quality if the objective is to enroll patients with left ventricular hypertrophy. Moreover, a given level of quality needs to be balanced with the feasibility, costs and risks to obtain it. Finally, an absolute vision of the quality must not lead to research conditions so far removed from everyday practice that the transposability of the research results could be compromised. Therefore, a more operational definition should be proposed: quality data is data that appropriately responds to the objectives for its use.

Consequently, we have defined quality data not only as data correctly representing reality, but above all as data that generates quality information, in a defined context, that allows the right decision to be taken at the right time.

\subsection{Quality criteria}

Tens of quality criteria have been proposed in the literature on health or in the general area of quality. To be concise, three fields of quality (data, information, process) can be distinguished and a certain number of criteria can be listed for each of them. The three 


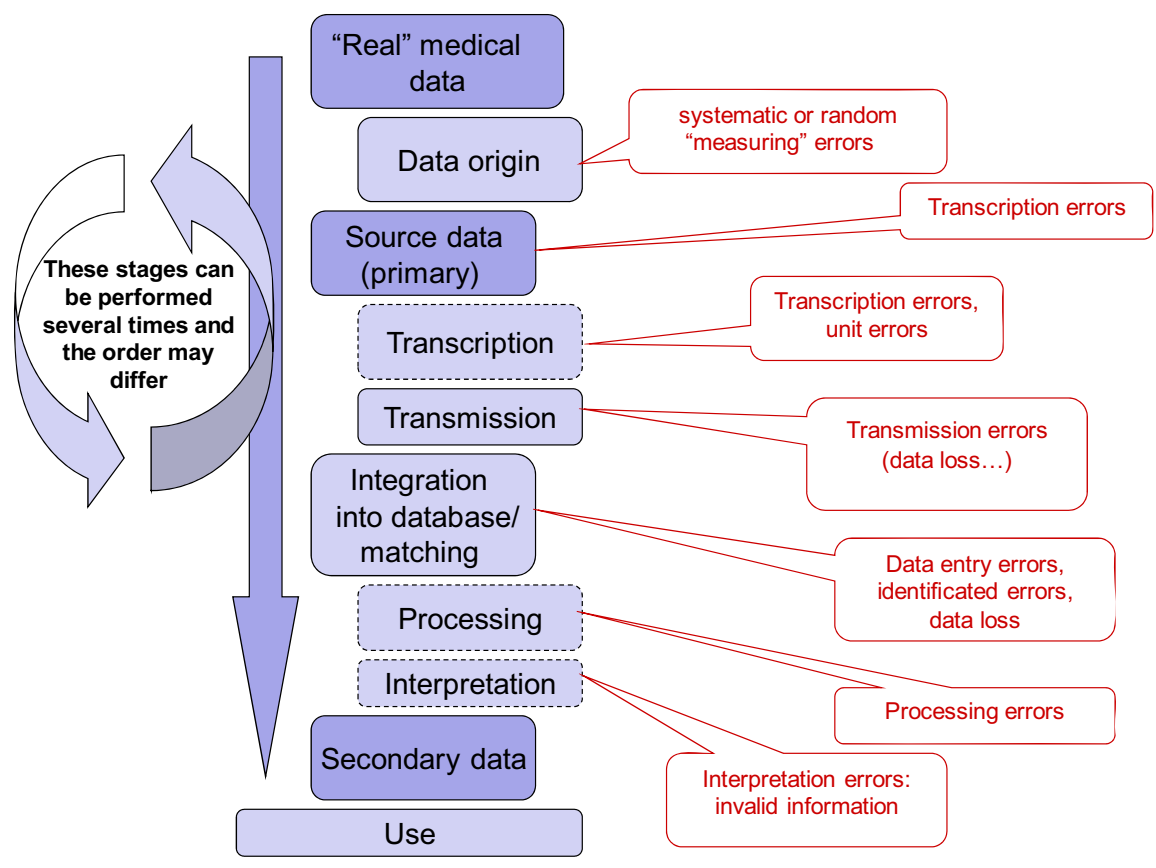

Fig. 1. The data chain: different stages and examples of errors likely to occur.

criteria of data quality that we have retained are validity (or accuracy), precision and reliability. The criteria of information quality (judged also in relation to its use) are, apart from the three previous criteria, the relevance (or usefulness or appropriateness), accessibility, exhaustivity, objectivity and interpretation. The criteria of quality of the process of obtaining information are: the cost-efficiency, absence of risks, confidentiality, security, durability, organization and verifiable (auditable) character of the information.

\section{The process and demonstration of critical points}

Initially, we described the stages from generating to using the data (figure 1). This representation is of course schematic and generic: the order of these stages may differ depending on the field and the study, and some stages may be performed several times. Then, for each stage, the main errors that could arise have been listed. For each error, the main causes have been identified.

The analysis is then focused on the data and critical stages. We have considered that the critical data were data for which quality (or lack of quality) poses a major risk problem. It mainly involves the primary endpoint, serious events, data modifying the benefit-risk ratio, etc. For this critical data, we have considered that the corresponding critical stages were those presenting an important problem for quality and for which the guidelines appear to be insufficiently detailed, unsuitable and/or imperfectly applied.

\section{Analysis of the existing information and the elaboration of recommendations}

In response to these issues and to ensure the quality of data, there are already many guidelines and procedures concerning data and data processing in all three fields. Examples include: the ICH guidelines for clinical trials ${ }^{[1]}$ or, more specifically, the Guideline on missing data in confirmatory clinical trials; ${ }^{[7]}$ the European Medicines Agency (EMA) guide on methodological standards in pharmacoepidemiology ${ }^{[8]}$ and the International Society of Pharmacoepidemiology (ISPE) guidelines for good pharmacoepidemiology practices ${ }^{[9]}$ for pharmacoepidemiology; and finally for pharmacovigilance, the new European guidelines of good pharmacovigilance practices. ${ }^{[10]}$

These guidelines and procedures are generally aimed at guaranteeing that database contents accurately reflect the source file. Many data management procedures exist. ${ }^{[1-13]}$ However, on analysis it becomes clear that these guidelines are insufficiently detailed on a critical and essential point: validation of the quality of source data and information in order to guarantee the validity/accuracy in the medical sense (e.g. is what is recorded as myocardial infarction is really myocardial infarction; what criteria were used for this diagnosis?). The existing guidelines do not mention (or hardly mention) these aspects, in particular the medical validity of source data. The latter is in fact implicitly considered as a gold standard and therefore cannot be questioned. Therefore, in the three fields, the data origin stage (i.e. formative stage of source data from assessment or observation) 


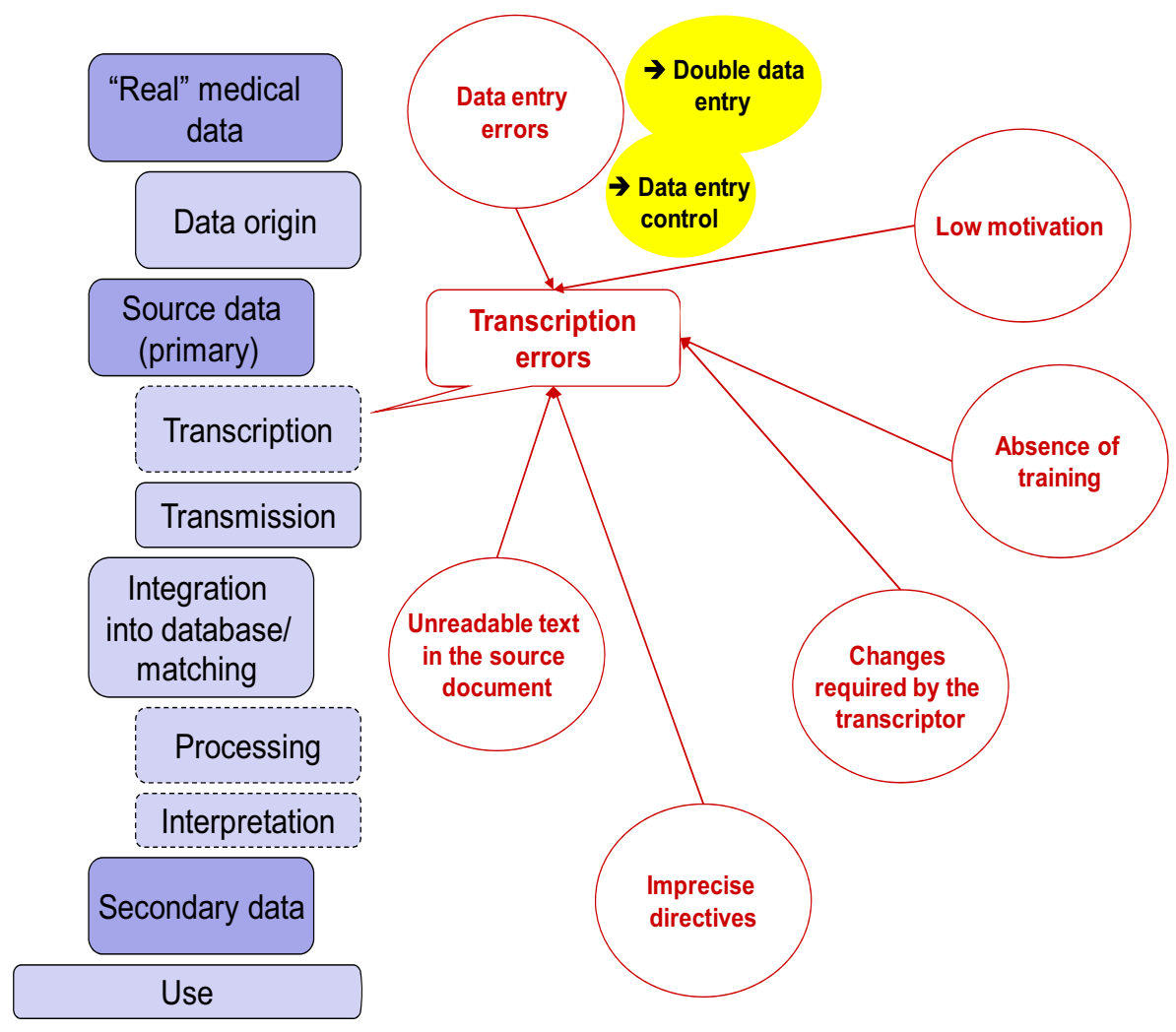

Fig. 2. Examples of reasons for certain types of errors and procedures for reducing them.

appears to be the priority. It is the quality of the initial medical diagnosis, but also the accuracy of the assessment methods (anthropometric, biological, electrocardiographic, etc.).

Other critical stages have been demonstrated in the chain of data processing, which depend on the field and data source (figure 1).

For clinical, biological or medico-administrative data, the origin of data (diagnostic error, incomplete medical record or record containing errors) and its transcription (errors, inappropriate coding, use of an imprecise code) represent major critical points. More specifically for medico-administrative data, if a risk of data loss or matching error exists during the following stages of transmission, processing and integration of data into databases, these errors are generally limited by the numerous existing procedures (yet little known to users).

For medico-administrative data, a second critical point is data interpretation. The interpretation of data during statistical analysis can be a source of errors. The main critical points are: the definition of the denominator (patients); the absence or imprecision of clinical data (clinical diagnosis, comorbidities, history, risk factors); the definition of treatment exposure. Further to the problem of quality, there is also the problem of use. Specific analysis techniques are required to take into consideration these limits. These issues are covered by several generic guidelines (e.g. the pharmacoepidemiology guidelines cited above) and other more specific ones. ${ }^{[14-16]}$ However, the literature is in agreement that they are insufficient. ${ }^{[17-20]}$

The quality of pharmacovigilance data is a shared concern. ${ }^{[21]}$ Several critical points have attracted our attention. The medical data is collected, transmitted and coded using the medical dictionnary for regulatory activities (MEDRA dictionary). This dictionary is complex and specific training is required in order to avoid variability in the coding process. Moreover, data interpretation for pharmacovigilance reporting is a major problem. Actually, the fact of an event being related or unrelated to a drug product has important consequences. It is therefore important that this "causality/imputability" data is validated after conferring with colleagues, without interpretation bias.

\section{Recommendations}

Finally, from the previously described analyses, the following recommendations were formulated according to a common principle (e.g. see figure 2). Some of these recommendations are original, whereas others are adapted from existing guidelines when the application needs reinforcing. 


\section{1. "Medical" data origin and transcription}

\subsubsection{Concerning the investigator}

- Certify the investigator: certification based on the informative quality of the medical data (officially approved training), with possible de-certification if the investigator has not fully respected the procedures and his obligations.

- Continue and reinforce professionalism in research: in particular, ideally have nurses as clinical research technicians in the department.

- Improve accessibility pharmacovigilance reporting (e.g. a web page for all vigilances).

- Adjust the investigator fees to the documentation and validation of the accuracy of the medical information collected, if appropriate.

\subsubsection{Concerning the origin, transcription and validation of critical data}

- Identify in the protocol the critical information that is compulsory and must be provided and validated by a physician and not simply under his supervision.

- Implement procedures adapted to the protocol that allow the quality of critical information to be reinforced (e.g. direct contact between clinical research associate [CRA]/investigator; outline in red the questions that must imperatively be filled in by the physician; procedure for reviewing records with the investigator to verify the veracity/accuracy and documentation of the data, etc.)

- Implement procedures for validating the critical information (e.g. role of "events physician" with the sponsor; systematic validation by an independent critical events evaluation committee).

- Respect good practices in metrology and psychometry for the development, validation and transcultural adaptations of assessment methods (in particular for assessing patient reported outcomes).

- Respect good laboratory practices, calibration procedures, and promote centralized review processes when appropriate.

- Simplify collection methods: restrict it to essential information (aim for quality and completeness of data rather than quantity of data and keep in perspective the final analysis that will be done).

- Improve the clarity and explicitation of instructions.

- Systematically use documents that have been translated (and validated) into the language of the investigator and patients, if appropriate.

- Standardize definitions of health conditions, assessment elements and their coding. Prepare professional thematic guidelines and/or consensus concerning the field of research and pharmacovigilance.

- Promote the use of electronic case report form (CRF) [that must respect the criteria of parsimony in terms of the amount of data collected].

- Implement procedures for real-time detection of abnormal variations in clinical or biological data (e-CRF or laboratory alerts).

- Focus the attention of those involved in pharmacovigilance on serious events (in the regulatory sense of the term).

- Standardize codifications (especially MedDRA), especially avoiding multiplying codes for the same medical entities.

- Include in the patient consent form an agreement that they can be contacted and/or the health establishment and treating physician can be contacted in order to complete pharmacovigilance or follow-up information (especially if the patient is lost to follow-up).

\subsection{Data processing}

- Validation (for critical data) of definitions and algorithms used.

- Explain the data management procedure (especially for medico-administrative databases).

- Develop control procedures for internal and external coherences.

\subsection{Reporting/publication}

- Formalize describing methods and results in the reports, especially in publications, to highlight the quality of data (further information on this point is provided by the consolidated standard of reporting trials [CONSORT], strenghtening the reporting of observational studies in epidemiology [STROBE] checklists, good research for comparatuve effectiveness [GRACE], ISPE and others checklists).

\section{Conclusion}

After reviewing the data chain in the various fields and for different sources used, we have attempted to provide solutions aimed at improving the quality of medical information generated from these data. Although the fields are different, the problems identified are similar.

Certain procedures for ensuring quality are already mentioned in current guidelines but should be further integrated, understood and applied in practice. This observation and these proposals could be used to elaborate simple and applicable procedures focused on 
the essential issue of improving medical information generated from clinical trials, pharmacoepidemiology studies and pharmacovigilance data.

\section{Conflicts of interests. None.}

Abbreviations. CONSORT: consolidated standard of reporting trials; CRA: clinical research associate; CRF: case report form; EMA: European Medicines Agency; GRACE: good research for comparative effectiveness; ICH: international conference of harmonization; ISPE: International Society of Pharmacology; MA: marketing authorization; MEDRA (dictionnary): medical dictionnary for regulatory activities; PMSI: french national discharge database (programme de médicalisation des systèmes d'information); RMI: resonnance magnetic imaging; SNIIRAM: french national Health insurance information system (système national d'informations inter régions d'Assurance maladie); STROBE: strengthening the reporting of observtional studies in epidemiology; SUSAR: suspected unexpected serious adverse reactions.

\section{References}

1. ICH. Guideline for good clinical practice E6 (R1), 1996. http://www.ich.org/fileadmin/Public_Web_Site/ICH_Products/Guidelines/Efficacy/E6_R1/Step4/E6_R1_Guideline.pdf

2. Näslund U, Gript L, Fischer-Hansen J, et al. The impact of an end-point committee in a large multicenter, randomized, placebo-controlled clinical trial: results with and without the end-point committee's final decision on endpoints. Eur Heart J 1999; 20(10): 771-7

3. Heagerty A, Deverly A, Palmer C, et al. The role of the critical event committee in a major cardiovascular outcome study. Blood Press 2002; 11(6) 339-44

4. Mahaffey KW, Roe MT, Dyke CK, et al. Misreporting of myocardial infarction end points: results of adjudication by a central clinical events committee in the PARAGON-B trial. Second Platelet IIb/IIIa Antagonist for the Reduction of Acute Coronary Syndrome Events in a Global Organization Network Trial. Am Heart J 2002; 143(2): 242-8

5. Proposition de règlement du Parlement européen et du Conseil relatif aux essais cliniques de médicaments à usage humain et abrogeant la directive 2001/20/CE. 2012. http://www.senat.fr/europe/textes_europeens/e7534.pdf

6. Strom BL, Kimmel SE, Hennessy S. Pharmacoepidemiology. Fifth edition. Oxford (UK): John Wiley \& Sons 2012

7. European Medicines Agency. Guideline on missing data in confirmatory clinical trials. EMA/CPMP/EWP/1776/99 Rev. 1, 2010 http://www.ema.europa.eu/docs/en_GB/document_library/ Scientific_guideline/2010/09/WC500096793.pdf
8. European Medicines Agency. The European Network of Centres for Pharmacoepidemiology and Pharmacovigilance (ENCePP). Guide on methodological standards in pharmacoepidemiology (Revision 1). 2012 EMA/95098/ 2010. http://www.encepp.eu/standards_and_guidances/documents/ ENCePPGuideofMethStandardsinPE.pdf

9. International Society for Pharmacoepidemiology. Guidelines for Good Pharmacoepidemiology Practices. ISPE. Revision: April 2007. http://www.pharmacoepi.org/resources/guidelines_08027.cfm

10. European Medicines Agency. Guideline on good pharmacovigilance practices (GVP) Module I - Pharmacovigilance systems and their quality systems. EMA/541760/2011. 2012. http://www.ema.europa.eu/docs/en_GB/ document_library/Scientific_guideline/2012/06/WC500129132.pdf

11. European Medicines Agency. ICH Topic E 6 (R1) CPMP/ICH/135/95. EMEA London. Guideline for Good Clinical Practice. 2002. http://www.edctp.org/fileadmin/documents/EMEA_ICH GCP_Guidelines_July_2002.pdf

12. Directive 2001/20/EC. Official Journal of the European Communities, L121/34, Luxembourg. 2001. http://eur-lex.europa.eu/LexUriServ/LexUriServ.do?uri=OJ:L:2001:121:0034:0044:en:PDF

13. Ohmann C, Kuchinke W, Canham S, et al. Standard requirements for GCPcompliant data management in multinational clinical trials. Trials 2011; 22(12): 85

14. Hall GC, Sauer B, Bourke A, et al. Guidelines for good database selection and use in pharmacoepidemiology research. Pharmacoepidemiol Drug Saf 2012; 21(1): 1-10

15. GPS - Good practice in secondary data analysis: revision after fundamental reworking. Version 2, January 2008. http://dgepi.de/fileadmin/pdf/leitlinien/gps-version2-final_ENG.pdf

16. The ISPOR good research practices for retrospective database analysis task force report - Part II. Value in Health 2009; 12(8): 1053-61. http://www.ispor.org/TaskForces/documents/RDPartll.pdf

17. Benchimol EI, Manuel DG, To T, et al. Development and use of reporting guidelines for assessing the quality of validation studies of health administrative data. J Clin Epidemiol 2011; 64(8): 821-9

18. Van Walraven C, Bennett C, Forster AJ. Administrative database research infrequently used validated diagnostic or procedural codes. J Clin Epidemiol 2011; 64(10): 1054-9

19. De Coster C, Quan H, Finlayson A, et al. Identifying priorities in methodological research using ICD-9-CM and ICD-10 administrative data: report from an international consortium. BMC Health Serv Res 2006; 15 (6): 77

20. Schneeweiss S, Avorn J. A review of uses of health care utilization databases for epidemiologic research on therapeutics. J Clin Epidemiol 2005; 58(4): 323-37

21. Lindquist M. Data quality management in pharmacovigilance. Drug Saf 2004; 27(12): 857-70

Correspondence and offprints: François Alla, Centre d'Épidémiologie clinique, Inserm-CHU de Nancy, Allée du Morvan, 54500 Vandœuvre-lesNancy, France.

E-mail: f.alla@chu-nancy.fr 